\title{
On the Heisenberg and Schrödinger Pictures
}

\author{
Shigeji Fujita', James MacNabb III'1, Akira Suzuki² \\ ${ }^{1}$ Department of Physics, University at Buffalo, State University of New York, Buffalo, USA \\ ${ }^{2}$ Department of Physics, Faculty of Science, Tokyo University of Science, Tokyo, Japan \\ Email: asuzuki@rs.kagu.tus.ac.jp
}

Received 9 December 2013; revised 8 January 2014; accepted 3 February 2014

Copyright (C) 2014 by authors and Scientific Research Publishing Inc.

This work is licensed under the Creative Commons Attribution International License (CC BY).

http://creativecommons.org/licenses/by/4.0/

(c) (i) Open Access

\begin{abstract}
A quantum theory for a one-electron system can be developed in either Heisenberg picture or Schrödinger picture. For a many-electron system, a theory must be developed in the Heisenberg picture, and the indistinguishability and Pauli's exclusion principle must be incorporated. The hydrogen atom energy levels are obtained by solving the Schrödinger energy eigenvalue equation, which is the most significant result obtained in the Schrödinger picture. Both boson and fermion field equations are nonlinear in the presence of a pair interaction.
\end{abstract}

\section{Keywords}

Heisenberg and Schrödingier Pictures; Many-Particle Systems; Indistinguishability; Second Quantization; Pauli's Exclusion Principle

\section{Introduction}

Both Heisenberg (HP) and Schrödinger pictures (SP) are used in quantum theory. Schrödinger solved Schrödinger eigenvalue equation for a hydrogen atom, and obtained the atomic energy levels. Heisenberg discussed the uncertainty principle based on the fundamental commutation relations. Both pictures are equivalent in dealing with a one-electron system. In dealing with many electrons or many photons a theory must be developed in the HP, incorporating the indistinguishability and Pauli's exclusion principle. A quantum theory must give a classical result in some limit. We will see that this limit is represented by $\hbar \rightarrow 0$. The HP, and not the SP, give the correct results for a many-particle system. The quantum field equation is nonlinear if a pair interaction exists.

\section{One Electron Systems}

We consider an electron in a potential energy field $V(\boldsymbol{r})$, where $\boldsymbol{r}$ is a position vector. In the Cartesian 
representation

$$
\boldsymbol{r}=(x, y, z) \equiv\left(q_{1}, q_{2}, q_{3}\right)
$$

The canonical momentum $\boldsymbol{p}$ is

$$
\boldsymbol{p}=\left(p_{x}, p_{y}, p_{z}\right) \equiv\left(p_{1}, p_{2}, p_{3}\right) .
$$

The Hamiltonian $H$ of the system is

$$
H=p^{2} /(2 m)+V(\boldsymbol{r}) .
$$

In the $\mathrm{HP}$ the coordinates and momenta $(q, p)$ are regarded as Hermitean operators satisfying the fundamental commutation relations (quantum conditions):

$$
\left[q_{j}, p_{k}\right] \equiv q_{j} p_{k}-p_{k} q_{j}=i \hbar \delta_{j k},\left[q_{j}, q_{k}\right]=\left[p_{j}, p_{k}\right]=0,
$$

where $\delta_{j k}$ is Kronecker's delta

$$
\delta_{j k}= \begin{cases}1 & \text { if } j=k \\ 0 & \text { if } j \neq k\end{cases}
$$

and $\hbar \equiv h /(2 \pi), h \equiv$ Planck constant.

The equations of motion for $q_{j}$ and $p_{j}$ are

$$
\frac{\mathrm{d} q_{j}}{\mathrm{~d} t}=\frac{1}{\mathrm{i} \hbar}\left[q_{j}, H\right], \quad \frac{\mathrm{d} p_{j}}{\mathrm{~d} t}=\frac{1}{\mathrm{i} \hbar}\left[p_{j}, H\right], j=1,2,3 .
$$

The two equations can be included in a single equation:

$$
\frac{\mathrm{d} \xi}{\mathrm{d} t}=\frac{1}{\mathrm{i} \hbar}[\xi, H],
$$

where $\xi$ represent any physical observable made out of the components of the position $\boldsymbol{r}=(x, y, z)$ and momentum $\boldsymbol{p}=\left(p_{x}, p_{y}, p_{z}\right)$. The angular momentum $\boldsymbol{I}=\boldsymbol{r} \times \boldsymbol{p}=\left(l_{x}, l_{y}, l_{z}\right)$ can be included also. Dirac has shown that in the small $\hbar$ limit:

$$
\frac{1}{\mathrm{i} \hbar}\left[q_{j}, p_{k}\right] \rightarrow\left\{q_{j}, p_{k}\right\} \text { as } \hbar \rightarrow 0,
$$

where

$$
\{A, B\} \equiv \sum_{j}\left\{\frac{\partial A}{\partial q_{j}} \frac{\partial B}{\partial p_{j}}-\frac{\partial B}{\partial q_{j}} \frac{\partial A}{\partial p_{j}}\right\}
$$

is the classical Poisson brackets.

In the SP we use the equivalence relations:

$$
p_{j}=-\mathrm{i} \hbar \frac{\partial}{\partial q_{j}}
$$

and write down the Schrödinger wave equation as

$$
\begin{aligned}
\mathrm{i} \hbar \frac{\partial \psi(\boldsymbol{r}, t)}{\partial t} & =H \psi(\boldsymbol{r}, t) \\
& =\left[-\frac{\hbar^{2}}{2 m}\left(\frac{\partial^{2}}{\partial x^{2}}+\frac{\partial^{2}}{\partial y^{2}}+\frac{\partial^{2}}{\partial z^{2}}\right)+V(x, y, z)\right] \psi(x, y, z, t) .
\end{aligned}
$$

The function $\psi$ is called the wave function. Normally, it is normalized such that

$$
\int_{\Omega} \mathrm{d}^{3} r \psi^{*}(\boldsymbol{r}, t) \psi(\boldsymbol{r}, t)=1
$$


where $\Omega$ is a normalization volume. The quantum average of an observable $\xi(\boldsymbol{r}, \boldsymbol{p})$ is defined by

$$
\langle\xi\rangle=\int \mathrm{d}^{3} r \psi^{*}(\boldsymbol{r}, t) \xi(\boldsymbol{r},-\mathrm{i} \hbar \nabla) \psi(\boldsymbol{r}, t) .
$$

If we use Dirac's ket and bra notations, then we can see the theoretical structures more compactly [1]. The quantum state $\psi$ is represented by the ket vector $|\psi, t\rangle$ or the bra vector $\langle\psi, t| \equiv(|\psi, t\rangle)^{\dagger}$. The Schrödinger equation of motion is

$$
\mathrm{i} \hbar \frac{\mathrm{d}}{\mathrm{d} t}|\psi, t\rangle=H|\psi, t\rangle
$$

whose Hermitean conjugate is

$$
-\mathrm{i} \hbar \frac{\mathrm{d}}{\mathrm{d} t}\langle\psi, t|=\langle\psi, t| H
$$

If we use the position representation and write

$$
\langle\boldsymbol{r} \mid \psi, t\rangle=\psi(\boldsymbol{r}, t)
$$

then we obtain Equation (11) from Equation (14).

We introduce the density operator $\rho$ defined by

$$
\rho(t) \equiv|\psi, t\rangle\langle\psi, t| .
$$

Using Equations (14) and (15), we obtain

$$
\mathrm{i} \hbar \frac{\mathrm{d} \rho}{\mathrm{d} t}=-[\rho, H] .
$$

This equation, called the quantum Liouville equation, has a reversed sign compared with the equation of motion for $\xi$, see Equation (7).

We can express the quantum average $\langle\xi\rangle$ of an observable $\xi$ as

$$
\langle\xi\rangle=\operatorname{tr}\{\xi \rho\}=\operatorname{tr}\{\rho \xi\},
$$

where $\operatorname{tr}$ denotes a one-particle trace. Operators under a trace commute.

We assume that the Hamiltonian $H$ in Equation (11) is a constant of motion. Then, Equation (14) can be reduced to the energy $(E)$ eigenvalue equation:

$$
H \psi_{E}(\boldsymbol{r})=\left[-\frac{\hbar^{2}}{2 m} \nabla^{2}+V(\boldsymbol{r})\right] \psi_{E}(\boldsymbol{r})=E \psi_{E}(\boldsymbol{r})
$$

after using a separation of variable method for solving Equation (11). Equation (20) is known as the Schrödinger energy-eigenvalue equation. The hydrogen atom energy-levels can be obtained from Equation (20) with $V(\boldsymbol{r})=-\mathrm{e}^{2} / 4 \pi \varepsilon_{0} r, \quad \varepsilon_{0}=$ permittivity.

Except for simple systems such as free electrons and simple harmonic oscillators, the Heisenberg equation of motion (7) [or the quantum Liouville Equation (18)] are harder to solve. This is so because the numbers of unknowns in the $n \times n$ matrix are more numerous than in the $1 \times n$ vector.

\section{Difficulties with the SP}

The following items have difficulties in the SP. They cannot be addressed properly.

(a) The Classical Mechanical Limit $(\hbar \rightarrow 0)$

Dirac showed that the fundamental commutation relations (8) can also be applied to a many particle system only if the Cartesian coordinates and momenta are used. The equation of motion (7) in the HP can be reduced to the classical equation of motion:

$$
\frac{\mathrm{d} \xi}{\mathrm{d} t}=\{\xi, H\} \quad \text { (classical) }
$$

in the classical limit 


$$
\hbar \rightarrow 0 \text {. }
$$

The Schrödinger equation of motion (11) does not have such a simple limit.

(b) Indistinguishability

All electrons are identical (indistinguishable) to each other. This is known as the indistinguishability. This property can be stated as follows:

Consider a system of $N$ electrons interacting with each other characterized by the Hamiltonian:

$$
H=\sum_{j=1}^{N} \varepsilon^{(j)}+\frac{1}{2} \sum_{j \neq k} \phi^{(j k)},
$$

where $\varepsilon^{(j)}$ is the kinetic energy and $\phi^{(j k)} \equiv \phi\left(\left|\boldsymbol{r}_{j}-\boldsymbol{r}_{k}\right|\right)$ is the pair interaction energy. Here the upper indices $j$ and $k$ denote the electrons. The indistinguishability requires that

$$
[\mathcal{P}, H]=0 \text { for all } \mathcal{P},
$$

where $\mathcal{P}$ are the permutation operators. For a three-particle system the permutation operators are

$$
\left(\begin{array}{lll}
1 & 2 & 3 \\
1 & 2 & 3
\end{array}\right),\left(\begin{array}{lll}
1 & 2 & 3 \\
1 & 3 & 2
\end{array}\right),\left(\begin{array}{lll}
1 & 2 & 3 \\
3 & 2 & 1
\end{array}\right),\left(\begin{array}{lll}
1 & 2 & 3 \\
2 & 1 & 3
\end{array}\right),\left(\begin{array}{lll}
1 & 2 & 3 \\
2 & 3 & 1
\end{array}\right),\left(\begin{array}{lll}
1 & 2 & 3 \\
3 & 1 & 2
\end{array}\right)
$$

The order of the permutation group for an $N$-particle system is $N$ !. The total momentum, the total angular momentum, and the total mass satisfy the same Equation (24). We may express this by

$$
[\mathcal{P}, X]=0, \quad X=\sum_{j=1}^{N} \xi^{(j)}
$$

(c) Boson Creation and Annihilation

Photons are bosons with full spin. They can be created and annihilated spontaneously. These processes can only be described by using creation and annihilation operators $\left(a, a^{\dagger}\right)$ both of which move, following the Heisenberg equations of motion. One can no more limit the number of bosons in the system.

(d) The Second Quantization for Bosons

Bosons can be treated using second-quantized operators $\left(a, a^{\dagger}\right)$ satisfying the Bose commutation rules:

$$
\left[a_{\mu}, a_{v}^{\dagger}\right] \equiv a_{\mu} a_{v}^{\dagger}-a_{v}^{\dagger} a_{\mu}=\delta_{\mu, v}, \quad\left[a_{\mu}, a_{v}\right]=\left[a_{\mu}^{\dagger}, a_{v}^{\dagger}\right]=0,
$$

where $(\mu, v)$ indicates particle states. Both operators $a$ and $a^{\dagger}$ move, following the Heisenberg equations of motion, e.g.

$$
\mathrm{i} \hbar \frac{\mathrm{d} a_{\mu}}{\mathrm{d} t}=\left[a_{\mu}, H\right]
$$

where $H$ is a many-boson Hamiltonian. The Hamiltonian for free photons is given by

$$
H=\sum_{p} \sum_{\sigma}\left(\frac{1}{2}+a_{p \sigma}^{\dagger} a_{p \sigma}\right) \hbar \omega_{p, \sigma},
$$

where $\omega_{p, \sigma}$ is angular frequency and $\sigma(=1$ or 2$)$ denotes the polarization indices.

(e) The Second Quantization for Fermions

Many fermions can be treated by using the complex dynamical operators $\left(c, c^{\dagger}\right)$ satisfying the Fermi anticommutation rules:

$$
\left[c_{\mu}, c_{v}^{\dagger}\right]_{+} \equiv c_{\mu} c_{\nu}^{\dagger}+c_{v}^{\dagger} c_{\mu}=\delta_{\mu, v}, \quad\left[c_{\mu}, c_{v}\right]_{+}=\left[c_{\mu}^{\dagger}, c_{v}^{\dagger}\right]_{+}=0 .
$$

Both operators $c$ and $c^{\dagger}$ move, following the Heisenberg equations of motion.

If the system contains many electrons, then we must consider Pauli's exclusion principle that no more than one fermion can occupy the same particle state. This is a restriction which cannot be described without considering permutation symmetry.

(f) Holes

Dirac showed [1] that there is symmetry between the occupied and unoccupied states for fermions, based on 
second quantization calculations. Holes are as much physical particles as electrons, and are fermions.

All six properties (a) - (f) can be discussed in the HP, but not in the SP. The last five (b) - (f) concern many-particle systems.

\section{Discussion}

We first discuss two relevant topics.

\section{(a) Wave packets}

Dirac assumed [1] that an experimentally observed particle correspond to a wave packet composed of the quantum waves, and showed that any wave packet moves obeying the classical mechanical laws of motion.

(b) The classical statistical limit

Free fermions (bosons) in equilibrium obey the Fermi (Bose) distribution law:

$$
f_{ \pm}(\varepsilon)=\frac{1}{\exp \left[(\varepsilon-\mu) / k_{B} T\right] \pm 1},
$$

where $\varepsilon$ is the kinetic energy, $k_{B}$ the Boltzmann constant, $T$ the absolute temperature and $\mu$ the chemical potential; the upper (lower) signes correspond to the Fermi (Bose) distribution functions. In the classical statistical limit, which is realized in either low density $(n)$ limit or high temperature $(T)$ limit, both distribution functions $f_{ \pm}(\varepsilon)$ approach the classical Boltzmann distribution function:

$$
\begin{gathered}
f_{B}(\varepsilon)=\exp \left[(\mu-\varepsilon) / k_{B} T\right], \\
f_{ \pm}(\varepsilon) \rightarrow f_{B}(\varepsilon) \text { as } T \rightarrow \infty \text { or } n \rightarrow 0 .
\end{gathered}
$$

For illustration we consider a free electron model for a metallic body-centred cubic (bcc) crystal such as sodium. Electrons subject to the exclusion principle are fermions which obey the Fermi distribution law. The heat capacity $C$ at the low temperatures $T$ shows a $T$-linear behavior. Phonons which are quanta of the lattice vibrations are bosons and they obey the Planck distribution law:

$$
f_{P}(\varepsilon)=\frac{1}{\exp \left(\varepsilon / k_{B} T\right)-1}
$$

since the chemical potential $\mu=0$ for phonons. The heat capacity arising from the phonons at low temperatures shows Debye $T^{3}$-law [2]. The electron wave packets have a linear size of the order of the lattice constant of the bcc crystal. The average phonon size is much greater, and is distributed with the Planck's law.

The effects of quantum statistics which involve $N$ ! permutations, see Equation (24), are much stronger than the effects of quantum entangling that grows linearly with $N$.

Let us introduce boson field operators $\psi(\boldsymbol{r}, t)$ and $\psi^{\dagger}(\boldsymbol{r}, t)$ which satisfy the Bose commutation rules:

$$
\left[\psi(\boldsymbol{r}), \psi^{\dagger}\left(\boldsymbol{r}^{\prime}\right)\right]=\delta^{(3)}\left(\boldsymbol{r}-\boldsymbol{r}^{\prime}\right), \quad\left[\psi(\boldsymbol{r}), \psi\left(\boldsymbol{r}^{\prime}\right)\right]=\left[\psi^{\dagger}(\boldsymbol{r}), \psi^{\dagger}\left(\boldsymbol{r}^{\prime}\right)\right]=0,
$$

where

$$
\text { (the time } t \text { omitted) }
$$

$$
\delta^{(3)}\left(\boldsymbol{r}-\boldsymbol{r}^{\prime}\right) \equiv \delta\left(x-x^{\prime}\right) \delta\left(y-y^{\prime}\right) \delta\left(z-z^{\prime}\right),
$$

and $\delta\left(x-x^{\prime}\right)$ is Dirac's delta-function.

A quantum many-boson Hamiltonian $H$ corresponding to the Hamiltonian in Equation (23) is

$$
H=\frac{\hbar^{2}}{2 m} \int \mathrm{d}^{3} r \nabla \psi^{\dagger}(\boldsymbol{r}) \cdot \nabla \psi(\boldsymbol{r})+\frac{1}{2} \int \mathrm{d}^{3} r \int \mathrm{d}^{3} r^{\prime} \psi^{\dagger}(\boldsymbol{r}) \psi^{\dagger}\left(\boldsymbol{r}^{\prime}\right) \phi\left(\left|\boldsymbol{r}-\boldsymbol{r}^{\prime}\right|\right) \psi\left(\boldsymbol{r}^{\prime}\right) \psi(\boldsymbol{r}),
$$

where $\psi\left(\psi^{\dagger}\right)$ are the field operators satisfying the equal-time commutation rules (35). The field equation is obtained from the Heisenberg Equation (28) as follows:

$$
\begin{aligned}
\mathrm{i} \hbar \frac{\partial \psi(\boldsymbol{r}, t)}{\partial t} & =[\psi(\boldsymbol{r}, t), H] \\
& =\int \mathrm{d}^{3} \boldsymbol{r} \boldsymbol{v} \cdot \nabla \psi(\boldsymbol{r}, t)+\int \mathrm{d}^{3} \boldsymbol{r}^{\prime} \phi\left(\left|\boldsymbol{r}-\boldsymbol{r}^{\prime}\right|\right) \psi^{\dagger}\left(\boldsymbol{r}^{\prime}, t\right) \psi\left(\boldsymbol{r}^{\prime}, t\right) \psi(\boldsymbol{r}, t),
\end{aligned}
$$


where $\boldsymbol{v} \equiv \partial \varepsilon / \partial \boldsymbol{p}$ is the velocity vector. We note that the field equation is nonlinear in the presence of a pair potential. This equation can be used to derive, and obtain the time dependent version of the Ginzburg-Landau (GL) equation [3]. This topic will be treated separately.

For a many-fermion system, fermion field operators $\psi_{F}(\boldsymbol{r}, t)$ and $\psi_{F}^{\dagger}(\boldsymbol{r}, t)$, satisfying the Fermi anticommutaion rules are introduced. The field equation is given by

$$
\begin{aligned}
\mathrm{i} \hbar \frac{\partial \psi_{F}(\boldsymbol{r}, t)}{\partial t}= & {\left[\psi_{F}(\boldsymbol{r}, t), H\right] } \\
= & \int \mathrm{d}^{3} r \boldsymbol{v} \cdot \nabla \psi_{F}(\boldsymbol{r}, t)+\frac{1}{2} \int \mathrm{d}^{3} \boldsymbol{r}^{\prime} \phi\left(\left|\boldsymbol{r}-\boldsymbol{r}^{\prime}\right|\right) \\
& \cdot\left[\psi_{F}(\boldsymbol{r}, t) \psi_{F}^{\dagger}\left(\boldsymbol{r}^{\prime}, t\right) \psi_{F}\left(\boldsymbol{r}^{\prime}, t\right)-\psi_{F}\left(\boldsymbol{r}^{\prime}, t\right) \psi_{F}^{\dagger}(\boldsymbol{r}, t) \psi_{F}(\boldsymbol{r}, t)\right] .
\end{aligned}
$$

This equation is also nonlinear in the presence of a pair potential $\phi^{(i k)}$.

\section{References}

[1] Dirac, P.A.M. (1958) The Principles of Quantum Mechanics. 4th Edition, Oxford Univ. Press, Oxford, 89-94; 121-125; 130-136; 136-139; 207-211; 227-237; 248-252.

[2] Debye, P. (1972) Annalen der Physik, 39, 789-839.

[3] Ginzburg, V.L. and Landau, L.D. (1950) Zh. Eksp. Teor. Fiz., 20, 1064-1082. [English translation in: L. D. Landau, “Collected papers,” Oxford: Pergamon Press (1965), 546.] 